\section{Houlin Liu}

Professor

e-mail: liuhoulin@ujs.edu.cn

Kai Wang ${ }^{1}$

Assistant Researcher

e-mail: wangkai@ujs.edu.cn

Shouqi Yuan

Professor

e-mail: Shouqiy@ujs.edu.cn

\section{Minggao Tan}

Associate Researcher

e-mail: tmgwxf@ujs.edu.cn

Yong Wang

Assistant Researcher e-mail: wylq@ujs.edu.cn

\section{Liang Dong \\ Assistant Researcher \\ e-mail: edongliang@hotmail.com}

National Research Center of Pumps and Pumping System Engineering and Technology, Jiangsu University, Zhenjiang, Jiangsu, 212013, P.R. China
Multicondition Optimization and Experimental Measurements of a Double-Blade Centrifugal Pump Impeller

In order to improve internal unsteady flow in a double-blade centrifugal pump (DBCP), this study used major geometric parameters of the original design as the initial values, heads at three conditions (i.e., 80\% design flow rate, 100\% design flow rate, and $120 \%$ design flow rate) as the constraints conditions, and the maximum of weighted average efficiency at the three conditions as the objective function. An adaptive simulated annealing algorithm was selected to solve the energy performance calculation model and the supertransitive approximation method was applied to fix optimal weight factors of individual objectives. On the basis of hydraulic performance optimization, three-condition automatic computational fluid dynamics (CFD) optimization of impeller meridional plane for the DBCP was realized by means of Isight software integrated Pro/E, Gambit, and Fluent software. The shroud arc radii $R_{0}$ and $R_{1}$, shroud angle $T_{1}$, hub arc radius $R_{2}$, and hub angle $T_{2}$ on the meridional plane were selected as the design variables and the maximum of weighted average hydraulic efficiency at the three conditions was chosen as the objective function. Performance characteristic test and particle image velocimetry (PIV) measurements of internal flow in the DBCP were conducted. Performance characteristic test results show that the weighted average efficiency of the impeller after the three-condition optimization has increased by $1.46 \%$ than that of original design. PIV measurements results show that vortex or recirculation phenomena in the impeller are distinctly improved under the three conditions. [DOI: 10.1115/1.4023077]

Keywords: double-blade centrifugal pump, multicondition optimization, energy performance, PIV measurement

\section{Introduction}

As one kind of nonclog sewage pumps [1], a double-blade centrifugal pump (DBCP) has the advantage of passage symmetry, good balance, and smooth operation. Therefore, DBCP is widely used to transport slurry, paper pulp, sewage, dirt, and other substances in paper manufacturing, sugar refining, chemical industry, metallurgy, mine, power plant, municipal construction, etc. $[2,3]$.

Current research on DBCP mainly focuses on hydraulic design and numerical simulation, while experimental research on the internal flow of DBCP is rare. Chen and Zhang [4] expounded design procedures of DBCP and proposed recommendations on the value of the area ratio between the impeller inlet section area and the outlet section area. Their prototype test results showed that the highest efficiency of the pump can reach 60\%. Huang [5] introduced the technical characteristics of DBCP impeller and gave some proposal to design impeller in the aspect of avoiding solid particles and fiber blocking. Zhao et al. [6] analyzed the influence of particle size on solid particle movement in the double-blade sewage pump, and the influence of initial particle concentration on pressure and solid phase distribution in the pump by using the computational fluid dynamics (CFD) method. Zhu et al. [7] optimized a DBCP impeller, and it was found that the efficiency of the optimization pump was obviously higher than existing domestic sewage pumps. Also, they analyzed the

\footnotetext{
${ }^{1}$ Corresponding author.

Manuscript received July 4, 2012; final manuscript received November 5, 2012; published online December 21, 2012. Assoc. Editor: Edward M. Bennett.
}

unsteady flow characteristics in a DBCP with the numerical simulation method, compared the static pressure and relative velocity distributions at different conditions, and analyzed the timedomain spectrums, frequency spectrums, and instant head [8,9].

Based on a traditional hydraulic design method, we have designed a DBCP with a specific speed of 116.7 [10,11]. The internal flow of the DBCP was measured with PIV, and the velocity distributions at seven different conditions from zero flow rate to 1.4 design flow rate were obtained. A vortex region was found in the impeller passage at the design condition, which undoubtedly caused hydraulic loss of the pump. It is thus necessary to develop some optimum method to improve the internal flow of DBCP, so as to provide theory foundation to design smooth operating and efficient DBCP.

At present, there are two methods to investigate the internal flow of centrifugal pumps. One is numerical simulation. With the development of computer fluids dynamics (CFD) and computer technique, CFD method has become a hot spot of internal flow of pump. Using CFD, one can study the unstable flows (e.g., rotating stall, secondary flow, and rotor-stator interaction) and cavitation characteristics of pumps [12-15]. The other one is experimental approach. As a powerful nonintrusive measuring technique that allows for measurement of instantaneous planar velocity fields, particle image velocimetry (PIV) has become attractive for investigations of complex internal flows in pumps [10,11,16-18].

In order to reduce hydraulic loss of a DBCP which was caused by the unsteady internal flow, this paper presents a multicondition optimum method on the basis of multicondition hydraulic performance optimization of impeller and multicondition automatic CFD optimization of impeller meridional plane. A DBCP with a specific speed of 116.7 was optimized with this method. 
Table 1 Main performance parameters of the investigated DBCP

\begin{tabular}{lcc}
\hline \hline Design flow rate $\left(\mathrm{m}^{3} \mathrm{~h}^{-1}\right)$ & $Q_{\mathrm{d}}$ & 34.48 \\
Head $(\mathrm{m})$ & $H$ & 4.44 \\
Rotational speed $(\mathrm{rpm})_{\text {Specific speed }^{\mathrm{a}}}$ & $n$ & 1000 \\
\hline
\end{tabular}

$\overline{{ }^{a} n_{\mathrm{s}}=3.65 n \sqrt{Q} / H^{3 / 4} \text {, where units of flow rate, rotational speed, and head }}$ are $\mathrm{m}^{3} / \mathrm{s}, \mathrm{rpm}$, and $\mathrm{m}$, respectively.

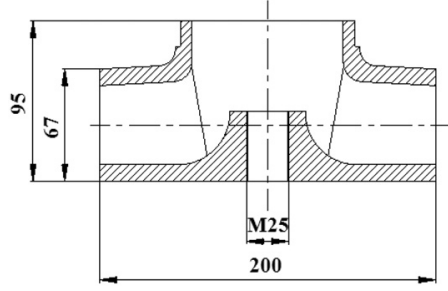

(a) Impeller meridional plane

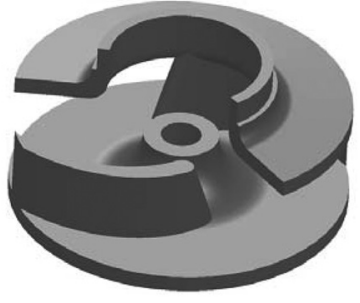

(b) Solid model
Fig. 1 Impeller model

Performance characteristic test and PIV measurements of internal flow in the DBCP were conducted.

\section{Original DBCP and Experiments}

2.1 Design Parameters. Main performance parameters of the investigated $\mathrm{DBCP}$ at the design condition are shown in Table 1.
2.2 DBCP Design. To improve antiwinding stability of the double-blade impeller, the blade inlet thickness is $15 \mathrm{~mm}$ and the blade outlet thickness is $7.5 \mathrm{~mm}$. Impeller meridional plane and solid model created using Pro/E are shown in Fig. 1. Impeller and volute are made of Perspex glass. The volute is designed by using the equivalent velocity moment method, whose cross section is a rectangle and type line is the logarithmic spiral. The DBCP semispiral suction chamber is made of stainless steel.

Main design parameters of the $\mathrm{DBCP}$ are as follows.

(1) Suction chamber. Inlet diameter $D_{\mathrm{s}}$ is $80 \mathrm{~mm}$.

(2) Impeller. Inlet diameter $D_{\mathrm{j}}$, outlet diameter $D_{2}$, outlet width $b_{2}$, blade outlet angle $\beta_{2}$, and blade wrap angle $\varphi$ are $90 \mathrm{~mm}, 200 \mathrm{~mm}, 47 \mathrm{~mm}, 30 \mathrm{deg}$, and $140 \mathrm{deg}$, respectively.

(3) Volute. Inlet diameter $D_{3}$, inlet width $b_{3}$, and outlet diameter $D_{\mathrm{d}}$ are $212 \mathrm{~mm}, 77 \mathrm{~mm}$, and $80 \mathrm{~mm}$, respectively.

2.3 Performance Characteristic Test. The performance characteristics test bench and the pump system are shown in Fig. 2. Main performance test equipment include a variable frequency control cabinet, a three-phase asynchronous motor, an electrical flowmeter, a pressure transmitter, a three-phase PWM digital power meter which was installed in the variable frequency control cabinet, etc. During the test, the motor speed is adjusted by the variable frequency control cabinet, the flow rate is measured by the electrical flowmeter, head is received by the mercury manometer, power is obtained by the three phase PWM digital power meter, and all of the experimental data are managed and analyzed by the data acquisition instrument.

Energy performance curve of $\mathrm{DBCP}$ at rotational speed $n=1000 \mathrm{rpm}$ is shown in Fig. 3. The test results show that the head of investigated DBCP at the design flow rate is $4.44 \mathrm{~m}$ and

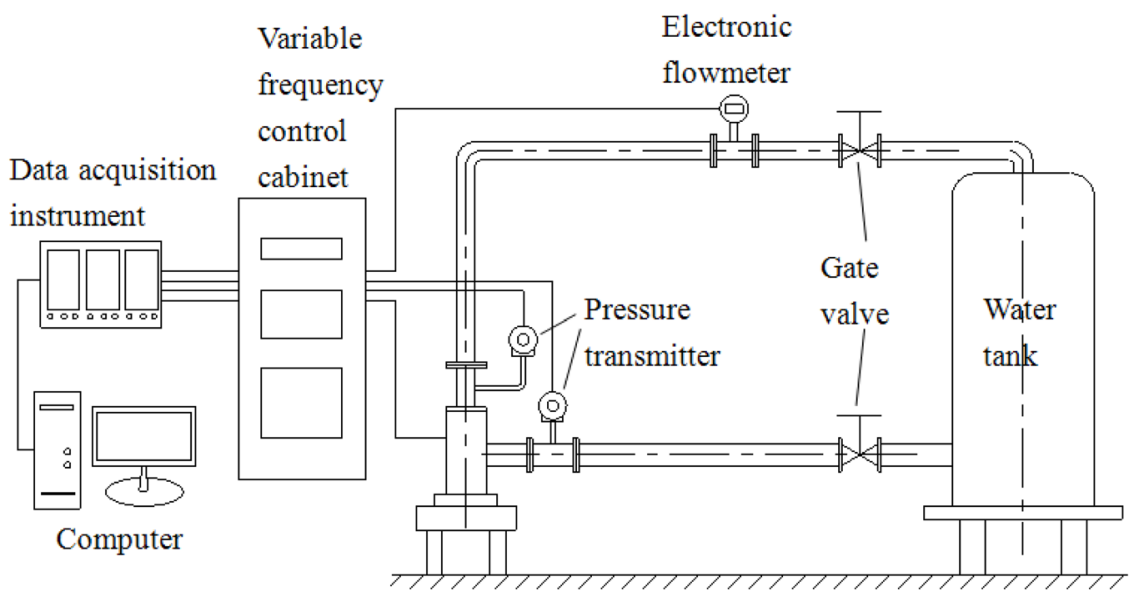

(a)

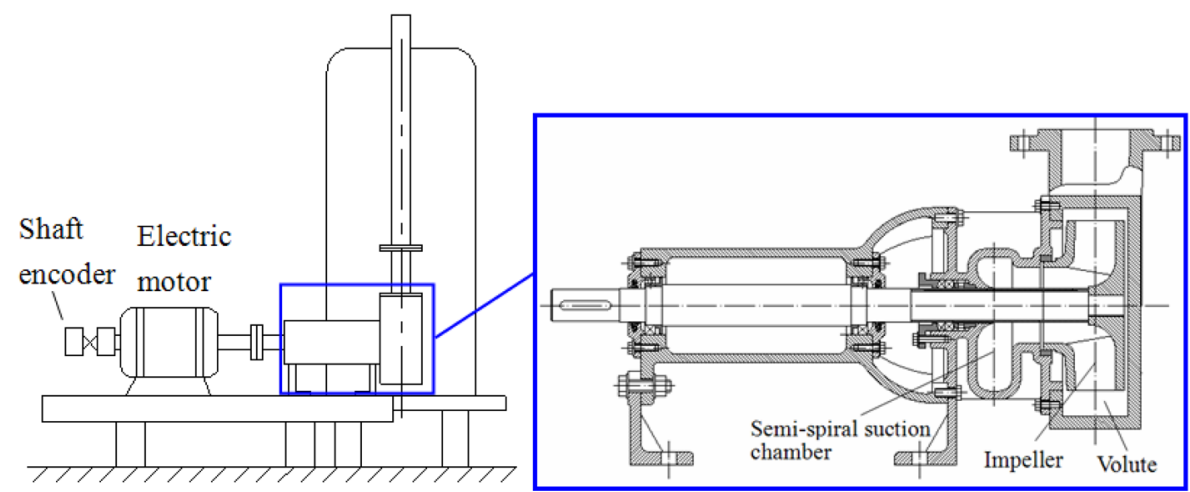

(b)

Fig. 2 Sketch of (a) test bench and (b) DBCP model 


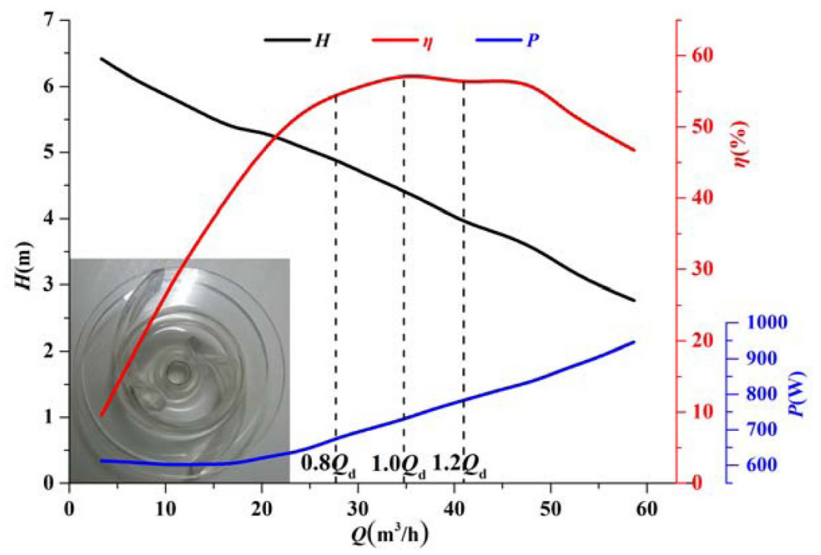

Fig. 3 Energy performance curve of DBCP

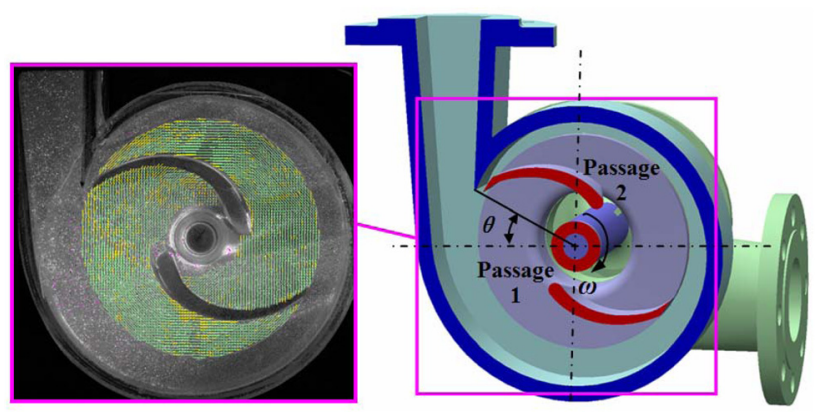

Fig. 4 Sketch of measurement region

its efficiency is $57.37 \%$, which is higher than the machinery industry standard of P.R. China (JB/T 8857-2000, 55.8\% pump efficiency is required).

2.4 PIV Measurements of Internal Flow for Impeller at the Design Flow Rate. The PIV system applied in this experiment is the TSI model. It mainly includes NewWave YAG200NWL pulse laser, the 610,035 laser pulse ${ }^{\mathrm{TM}}$ synchronizer, the 630,059 power view ${ }^{\mathrm{TM}}$ plus $4 \mathrm{M}$ PIV camera, Insight $3 \mathrm{G}$, the 610,015 light arm, light source lens, and so on.

The middle section of the impeller is selected as the investigation plane. The measurement region is comprised of the impeller passage 1 and passage 2, which is illustrated in Fig. 4. As seen from the figure, the angle between the blade suction surface near the tongue and the horizontal axis is $\theta=28 \mathrm{deg}$.

Since with PIV the displacement of particles and not of the flow itself is measured, it is required that the seeding particles are able to follow the flow. It is therefore essential that seeding particles are small enough to track the flow accurately and, on the other hand, are big enough to scatter sufficient light for the camera to detect them. Because $\mathrm{Al}_{2} \mathrm{O}_{3}$ powders, whose average diameter is $1 \mu \mathrm{m}$, can satisfy the above criteria and are relatively cheap, they are chosen as tracer particles in the test.

Because the test flow field is a closed rotating flow field, it is unable to put the calibration target into the pump. To solve the calibration problem, an equivalent calibration method is put forward, namely, putting the calibration target inside a calibration water tank, as shown in Fig. 5. The calibration water tank consists of a water tank and baffle. They are made of Perspex glass, which is the same material as the impeller and the volute. The thickness

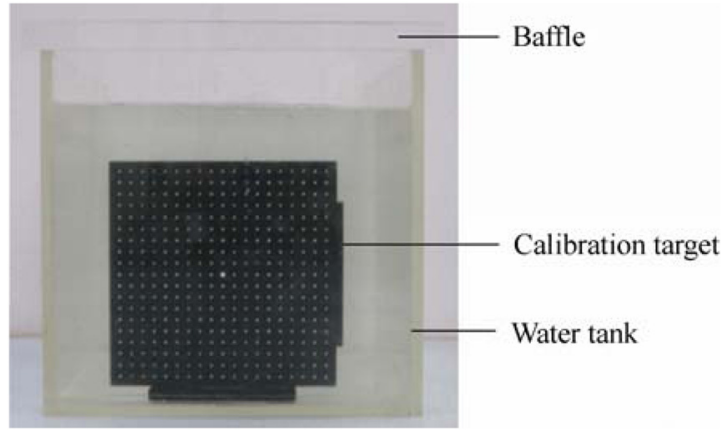

Fig. 5 Equivalent calibration

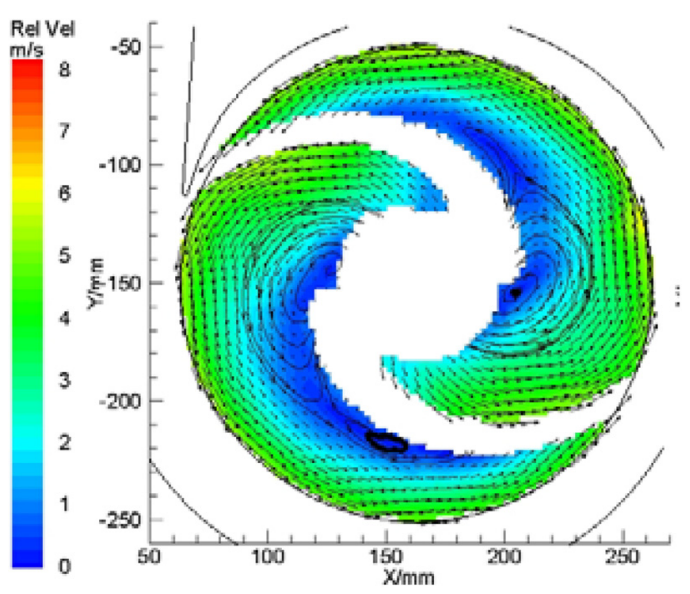

Fig. 6 Relative velocity distribution of impeller

of the water tank is equal to that of the volute, while the thickness of the baffle is equal to that of the impeller hub.

Figure 6 presents the relative velocity distribution inside of the DBCP impeller at $100 \% Q_{\mathrm{d}}$. There are vortex regions in the impeller passage 1 and passage 2 . Besides, there are recirculation phenomena in the blade inlet. The incurred unsteady flow would result in unfavorably higher hydraulic losses. Thus in this paper, multicondition optimization of the DBCP impeller was done on the basis of considering energy losses in the DBCP.

\section{Multicondition Optimization}

3.1 Multicondition Optimization Method. The multicondition optimization process is indicated in Fig. 7. Its main steps are as follows.

Step 1: Multicondition hydraulic performance optimization of impeller. Use major geometric parameters of original design as the initial values, heads at three conditions (i.e., $80 \% Q_{\mathrm{d}}$, $100 \% Q_{\mathrm{d}}$, and $\left.120 \% Q_{\mathrm{d}}\right)$ as the constraints conditions, and the maximum of weighted average efficiency at the three conditions as the objective function. The adaptive simulated annealing algorithm is selected to solve the energy performance calculation model and the supertransitive approximation method is used to fix optimal weight factors of individual objectives.

Step 2: Multicondition automatic CFD optimization of impeller meridional plane. On the basis of multicondition hydraulic performance optimization, the automatic CFD optimization of impeller meridional plane was performed using Isight software integrated Pro/E, Gambit, and Fluent software to realize multicondition. The shroud arc 


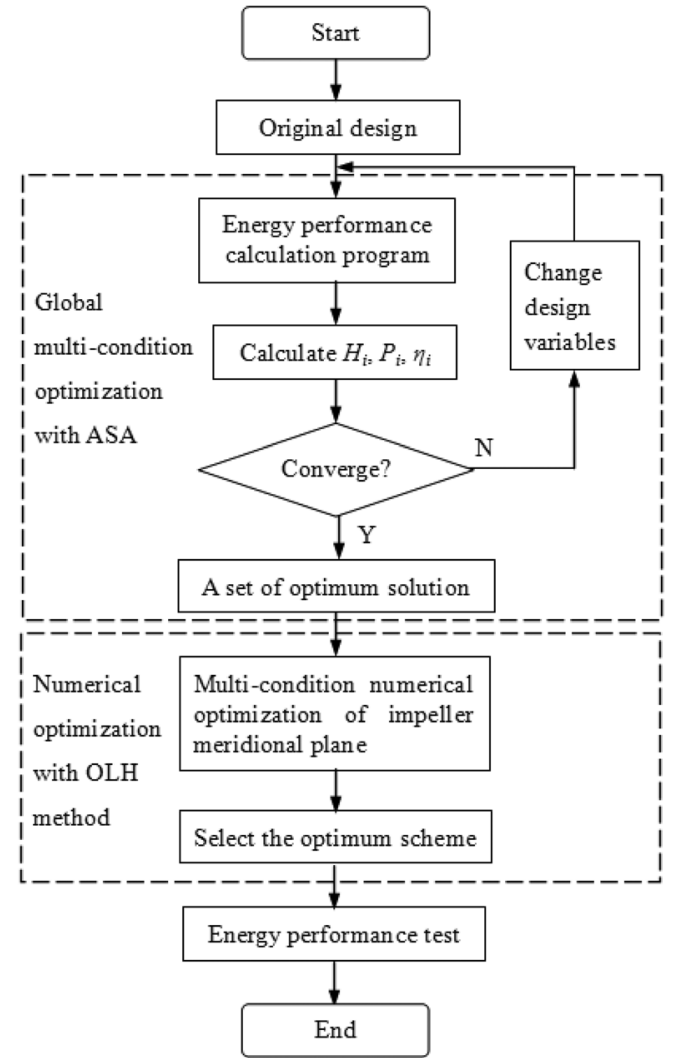

Fig. 7 Multicondition optimization process of DBCP

radii $R_{0}$ and $R_{1}$, shroud angle $T_{1}$, hub arc radius $R_{2}$, and hub angle $T_{2}$ on the meridional plane are selected as the design variables and the maximum of weighted average hydraulic efficiency at the three conditions is considered as the objective function.

Step 3: Experimental verification.

3.2 Multicondition Hydraulic Performance Optimization of Impeller

3.2.1 Multicondition Energy Performance Calculation Model. On the basis of energy losses in the centrifugal pump, the energy performance calculation model of centrifugal pumps for the whole flow rate range was established.

Leakage flow rate through the sealing ring at the single-stage centrifugal pump impeller inlet $q_{1}$ is calculated as follows:

$$
q_{1}=\mu f \sqrt{2 g \Delta H_{\mathrm{m}}}
$$

where $f$ is the sectional area of the sealing gap defined as $f=2 \pi R_{\mathrm{m}} \mathrm{b}, R_{\mathrm{m}}$ is the radius of the sealing ring, $b$ is the sealing gap width, $\mu$ is the velocity coefficient of the sealing gap defined as $\mu=1 / \sqrt{1+0.5 \zeta+(\lambda l / 2 b)}, \zeta$ is the coefficient of fillet radius with $\zeta=0.5$ to $0.9, \lambda$ is the hydraulic resistance coefficient with $\lambda=0.04$ to $0.06, l$ is the sealing gap length, and $\Delta H_{\mathrm{m}}$ is the pressure difference over the seal. $\Delta H_{\mathrm{m}}=0.6 H$ at $n_{\mathrm{s}} \leq 100$, while $\Delta H_{\mathrm{m}}=0.7 H$ at $n_{\mathrm{s}} \geq 100$.

Volumetric efficiency $\eta_{\mathrm{v}}$ is calculated as follows:

$$
\eta_{\mathrm{v}}=\frac{Q_{\mathrm{t}}-q_{1}}{Q_{\mathrm{t}}}
$$

Assuming $v_{u 1}=0$, the ideal head of the centrifugal pump for a finite number of blades is calculated as follows:

$$
H_{t}=\frac{\pi D_{2} n}{60 g}\left(\sigma \frac{\pi D_{2} n}{60}-\frac{Q}{\eta_{v} \pi D_{2} b_{2} \psi_{2} \tan \beta_{2}}\right)
$$

where $D_{2}$ is the impeller outlet diameter, $n$ is the rotational speed, $\sigma$ is the Stodola slip factor, $\sigma=1-(\pi / z) \sin \beta_{2}, z$ is the number of blades, $\beta_{2}$ is the blade outlet angle of the impeller, $Q$ is the flow rate of the pump, $b_{2}$ is the outlet blade width of the impeller, $\Psi_{2}$ is the blockage coefficient of the blade outlet, $\Psi_{2}=1-z S_{u 2} / D_{2} \pi$, and $S_{u 2}$ is the circumference thickness of the blade outlet.

Hydraulic losses in the pump are mainly caused by flow passage in the suction chamber, impeller, and volute, where those in the suction chamber are little and usually ignored.

Inlet incidence loss of the impeller $\Delta h_{1}$ is calculated as follows:

$$
\Delta h_{1}=k_{1} \frac{1}{g} u_{2}^{2}\left(\frac{D_{1}}{D_{2}}\right)^{2}\left(\frac{Q}{Q_{d}}-1\right)^{2}
$$

where $k_{1}$ is the loss coefficient which generally falls in the range of 0.7 to $0.9, D_{1}$ is the impeller inlet diameter, and $Q_{\mathrm{d}}$ is the flow rate at the design condition.

Friction loss in the impeller passage $\Delta h_{2}$ is calculated as follows:

$$
\Delta h_{2}=z \times \lambda_{1} \times \frac{l_{1 \mathrm{a}}}{D_{1 \mathrm{a}}} \times \frac{v_{1 \mathrm{a}}^{2}}{2 g}
$$

where $\lambda_{1}$ is the hydraulic resistance coefficient, which is defined as $\lambda_{1}=\left[1.74+2 \lg \left(D_{1 \mathrm{a}} / 2 \delta_{1}\right)\right]^{-2}, l_{1 a}$ is the length of impeller passage,

$$
l_{1 \mathrm{a}}=\frac{D_{j}+D_{2}}{4} \times \frac{\varphi}{180} \times \pi \times \frac{1}{2}\left(\frac{1}{\cos \beta_{1}}+\frac{1}{\cos \beta_{2}}\right)
$$

$v_{1 \mathrm{a}}$ is the average velocity in the impeller inlet area and outlet area, $v_{1 \mathrm{a}}=\left(v_{\mathrm{m} 1}+v_{\mathrm{m} 2}\right) / 2, D_{1 \mathrm{a}}$ is the hydraulic equivalent diameter, which is the calculated average value of the inlet hydraulic equivalent diameter and the outlet hydraulic equivalent diameter, $\delta_{1}$ is the surface roughness of the impeller, and $\varphi$ is the blade wrap angle of the impeller.

Diffuser loss in the impeller $\Delta h_{3}$ is calculated as follows:

$$
\Delta h_{3}=k_{3} \times \frac{\left|w_{1}^{2}-w_{2}^{2}\right|}{2 g}
$$

where $k_{3}$ is the loss coefficient, which ranges from 0.1 to 0.2 .

Hydraulic loss, which is produced by axial flow of the impeller inlet turning into radial flow $\Delta h_{4}$, is calculated as follows:

$$
\Delta h_{4}=k_{4} \frac{v^{2}}{2 g}
$$

where $k_{4}$ is the loss coefficient and $v$ is the inlet velocity of the impeller.

Hydraulic loss of the impeller outlet $\Delta h_{5}$ is calculated as follows:

$$
\Delta h_{5}=k_{5} \frac{v_{\mathrm{m} 2}^{2}+\left(v_{u_{2}}-v_{\mathrm{s}}\right)^{2}}{2 g}
$$

where $k_{5}$ is the loss coefficient defined as $k_{5}=B / \sqrt{\pi D_{2} b_{2} \psi_{2} \sin \beta_{2}}$, and $v_{\mathrm{s}}$ is the average velocity of the volute throat. 


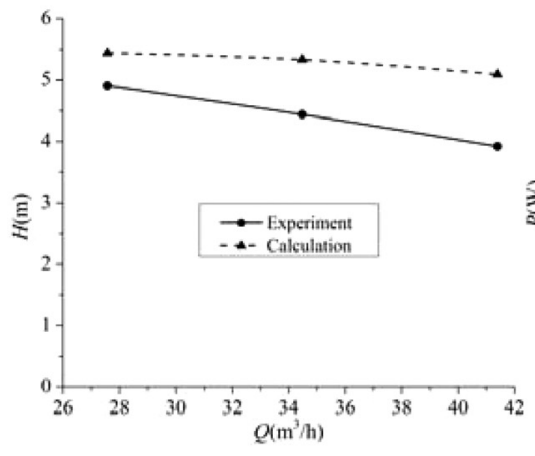

(a) Head

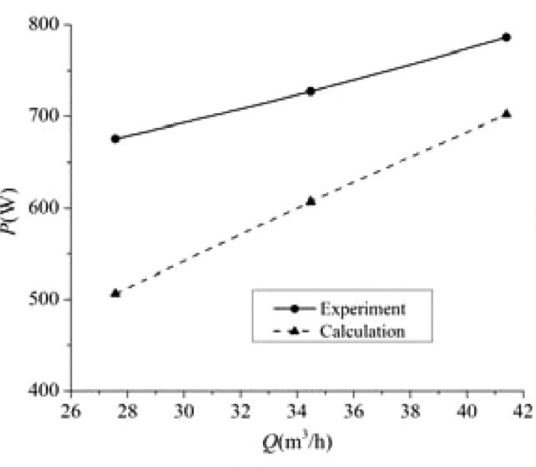

(b) Power

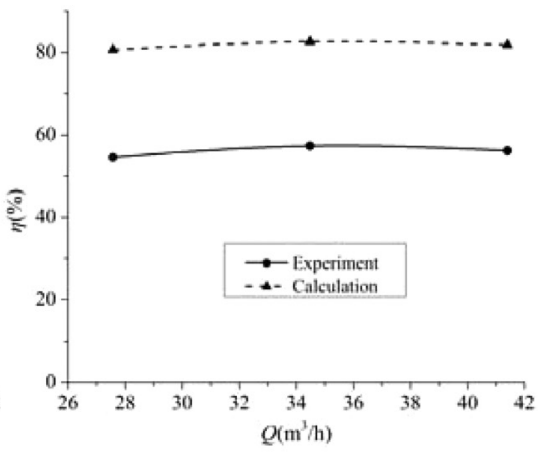

(c) Efficiency

Fig. 8 Energy performance comparison with calculation and experiment

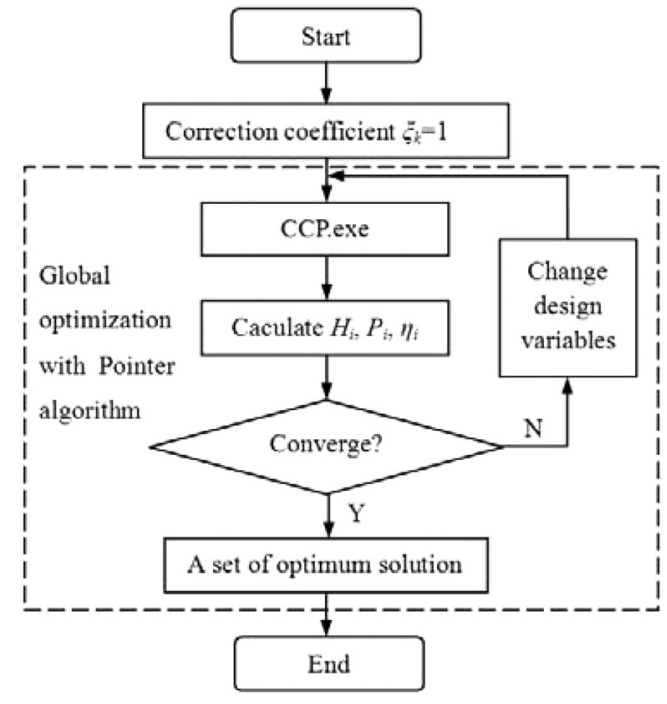

Fig. 9 Optimization process of correct coefficients

Friction loss in the volute passage $\Delta h_{6}$ is calculated as follows:

$$
\Delta h_{6}=\lambda_{2} \frac{l_{2 \mathrm{a}}}{D_{2 \mathrm{a}}} \frac{v_{\text {th }}}{2 g}
$$

where $\lambda_{2}=\left[1.2+2 \lg \left(D_{2 \mathrm{a}} / 2 \delta_{2}\right)\right]^{-2}$ is the hydraulic resistance coefficient, $l_{2 \mathrm{a}}=\pi\left(1-\varphi_{0} / 360\right)\left(D_{3}+D_{2 \mathrm{a}}\right)$ is the length of the volute passage, $v_{\text {th }}$ is the average velocity of the volute, $D_{2 a}=\sqrt{2 F_{t} / \pi}$ is the hydraulic equivalent diameter, $F_{\mathrm{t}}$ is the volute throat area, $D_{3}$ is the volute inlet diameter, $\varphi_{0}$ is the volute tongue angle, and $\delta_{2}$ is the surface roughness of the volute.

Diffuser loss in the volute $\Delta h_{7}$ is calculated as follows:

$$
\Delta h_{7}=k_{7} \frac{\left(v_{u 2}^{2}-v_{\mathrm{s}}^{2}\right)}{2 g}
$$

where $k_{7}$ is the loss coefficient, which ranges from 0.2 to 0.5 .
Table 3 Optimization results

\begin{tabular}{lccccccc}
\hline \hline & $D_{2} / \mathrm{mm}$ & $D_{1} / \mathrm{mm}$ & $\beta_{2} / \mathrm{deg}$ & $\beta_{1} / \mathrm{deg}$ & $b_{2} / \mathrm{mm}$ & $b_{1} / \mathrm{mm}$ & $\varphi_{1} / \mathrm{deg}$ \\
\hline Original & 200.0 & 91.0 & 30.0 & 15.1 & 47.0 & 57.0 & 140.0 \\
Optimization & 201.2 & 90.7 & 23.8 & 21.4 & 44.6 & 53.2 & 177.5 \\
\hline \hline
\end{tabular}

Therefore, the total hydraulic losses in pump $\Delta h$ are as follows:

$$
\Delta h=\sum_{j=1}^{7} \Delta h_{j}
$$

Actual head $H$ is as follows:

$$
H=H_{\mathrm{t}}-\Delta h
$$

Hydraulic efficiency $\eta_{\mathrm{h}}$ is as follows:

$$
\eta_{\mathrm{h}}=\frac{H}{H_{\mathrm{t}}}
$$

Mechanical friction losses in the bearing and stuffing box $\Delta P_{1}$ usually are $1 \%$ to $3 \%$ of shaft power.

Frick friction loss $\Delta P_{2}$ is as follows:

$$
\Delta P_{2}=1.1 \times 10^{-6} \rho u_{2}^{3} D_{2}\left(D_{2}+5 e\right) \times 75 \mathrm{~kW}
$$

where $e$ is the thickness of the impeller shroud and hub.

Shaft power $P$ is as follows:

$$
P=\rho g Q_{\mathrm{t}} H_{\mathrm{t}}+\Delta P_{1}+\Delta P_{2}
$$

Mechanical efficiency $\eta_{\mathrm{m}}$ is as follows:

\begin{tabular}{|c|c|c|c|c|c|c|c|c|c|c|c|c|}
\hline & $Q\left(\mathrm{~m}^{3} / \mathrm{h}\right)$ & $\xi_{0}$ & $\xi_{1}$ & $\xi_{2}$ & $\bar{\xi}_{3}$ & $\xi_{4}$ & $\xi_{5}$ & $\xi_{6}$ & $\xi_{7}$ & $\xi_{8}$ & $\xi_{9}$ & $\xi_{10}$ \\
\hline $80 \% Q_{\mathrm{d}}$ & 27.56 & 0.953 & 1.832 & 0.841 & 1.520 & 1.523 & 1.800 & 1.515 & 1.804 & 1.054 & 1.464 & 0.744 \\
\hline $100 \% Q_{\mathrm{d}}$ & 34.48 & 0.873 & 0.961 & 1.313 & 1.476 & 1.434 & 1.504 & 1.859 & 0.594 & 0.999 & 1.210 & 0.746 \\
\hline $120 \% Q_{\mathrm{d}}$ & 41.40 & 0.859 & 1.443 & 1.464 & 1.856 & 1.633 & 1.093 & 1.913 & 1.891 & 0.636 & 0.879 & 0.759 \\
\hline
\end{tabular}

$$
\eta_{\mathrm{m}}=\frac{\rho g Q_{\mathrm{t}} H_{\mathrm{t}}}{P}
$$

Total efficiency $\eta$ is as follows:

$$
\eta=\eta_{\mathrm{h}} \eta_{\mathrm{m}} \eta_{\mathrm{v}}
$$

Table 2 Loss correct coefficients 
Table 4 Grid number in grid independence test

\begin{tabular}{|c|c|c|c|c|c|c|c|c|c|}
\hline \multirow[b]{2}{*}{ Scheme } & \multicolumn{3}{|c|}{1} & \multicolumn{3}{|c|}{2} & \multicolumn{3}{|c|}{3} \\
\hline & Suction chamber & Impeller & Volute & Suction chamber & Impeller & Volute & Suction chamber & Impeller & Volute \\
\hline Interval size & 3.5 & 3 & 3.5 & 3 & 2.5 & 3 & 2.5 & 2 & 2.5 \\
\hline Grid number & 121,811 & 310,839 & 254,387 & 266,738 & 474,704 & 458,347 & 413,127 & 737,002 & 737,752 \\
\hline Total grid number & \multicolumn{3}{|c|}{687,037} & \multicolumn{3}{|c|}{$1,199,789$} & \multicolumn{3}{|c|}{$1,887,881$} \\
\hline
\end{tabular}

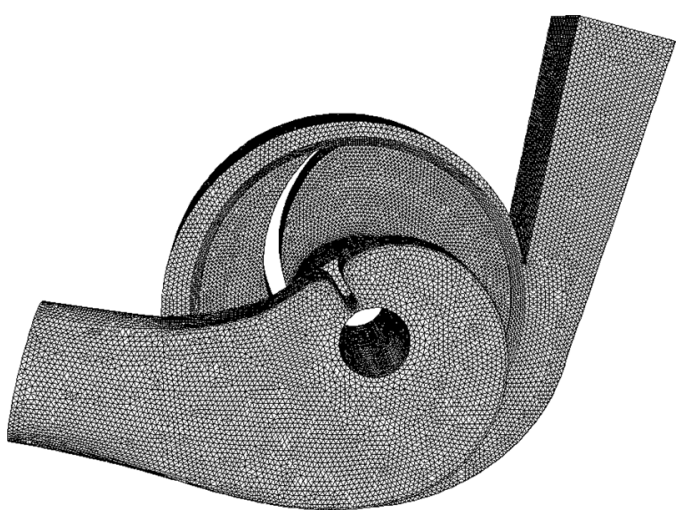

Fig. 10 Calculating mesh

Table 5 Results of grid independence test

\begin{tabular}{lrrr}
\hline \hline Scheme & 1 & \multicolumn{1}{c}{2} & \multicolumn{1}{c}{3} \\
\hline Head $H / \mathrm{m}$ & 4.22 & 4.30 & 4.31 \\
Relative error of head $\Delta H / \%$ & -4.09 & -3.15 & -2.93 \\
Efficiency $\eta / \%$ & 62.69 & 60.41 & 60.20 \\
Absolute error of efficiency $\delta \eta / \%$ & 5.37 & 3.09 & 2.88 \\
\hline \hline
\end{tabular}

3.2.2 Energy Performance Calculation. Values of the parameters used in the energy performance calculation model are as follows:

$R_{\mathrm{m}}=52 \mathrm{~mm}, \quad b=0.5 \mathrm{~mm}, \quad \zeta=0.7, \quad \lambda=0.05, \quad l=12 \mathrm{~mm}$. $k_{1}=0.7, k_{3}=0.1, k_{4}=0.21, k_{7}=0.2, \delta_{1}=0.1 \mathrm{~mm}, \delta_{2}=0.1 \mathrm{~mm}$, $\Delta P_{1}=2 \% \times P$, and $e=10 \mathrm{~mm}$.

Figure 8 shows the comparison of the energy performance obtained with calculation and experiment at $80 \% Q_{\mathrm{d}}, 100 \% Q_{\mathrm{d}}$, and $120 \% Q_{\mathrm{d}}$. It can be seen that compared with experimental data, the calculation errors of head and efficiency at the three conditions are much greater. So it is necessary to correct loss coefficients in the energy performance calculation model.

3.2.3 Loss Coefficients Correction. Energy performance calculation model with loss correct coefficients are as follows:

$$
H=\xi_{0} H_{\mathrm{t}}-\sum_{k=1}^{7} \xi_{k} \Delta h_{k}
$$

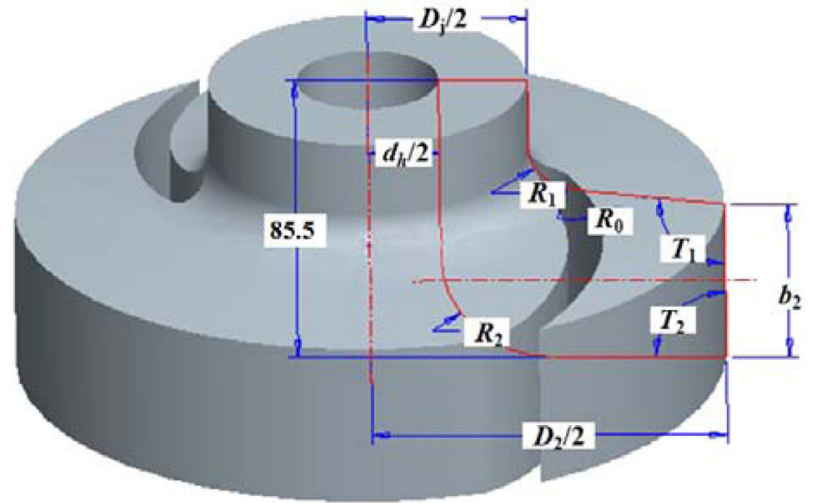

Fig. 11 Impeller meridional plane of DBCP

$$
\begin{gathered}
P=\rho g\left(Q+\xi_{8} q_{1}\right) \xi_{0} H_{t}+\xi_{9} \Delta P_{1}+\xi_{10} \Delta P_{2} \\
\eta=\frac{\rho g Q H}{P}
\end{gathered}
$$

where $\xi_{k}$ is the correct coefficient with $k=0,1, \ldots, 10$.

Figure 9 shows the optimization process of the correct coefficients. Optimization steps of correct coefficients are as follows.

Step 1: Assume initial values of each correct coefficient to be $\xi_{k}=1.0$.

Step 2: On the basis of Eqs. (18), (19), and (20), compile calculation program of correct coefficients CCP.exe with Visual $\mathrm{C}++2010$

Step 3: Select correct coefficients as design variables, head values at the three conditions as constraint and efficiency values at the three conditions as objective, and choose Pointer algorithm to solve correct coefficients in the Isight platform.

Step 4: If convergence criterion is not reached, change design variable values and repeat step 3 until convergence criterion has been reached.

Step 5: After convergence, a set of optimum correction coefficients will be obtained.

Corrected losses coefficients of DBCP at the three conditions are shown in Table 2.

3.2.4 Multicondition Hydraulic Optimization Model. Defining $\boldsymbol{x}=\left[D_{2}, \beta_{2}, b_{2}, D_{1}, \beta_{1}, b_{1}, \varphi_{1}\right]^{\mathrm{T}}$ yields

$$
\bar{\eta}=\frac{\sum \eta_{i}(\boldsymbol{x}) W_{i}}{\sum W_{i}} \rightarrow \max
$$

\begin{tabular}{|c|c|c|c|c|c|c|c|}
\hline & \multirow[b]{2}{*}{$Q / \mathrm{m}^{3} \mathrm{~h}^{-1}$} & \multicolumn{3}{|c|}{$H$} & \multicolumn{3}{|c|}{$\eta$} \\
\hline & & Experiment $/ \mathrm{m}$ & Numerical simulation/m & Relative error $/ \%$ & Experiment $/ \%$ & Numerical simulation/\% & Absolute error $/ \%$ \\
\hline $80 \% Q_{\mathrm{d}}$ & 27.58 & 4.91 & 4.75 & -3.26 & 54.56 & 55.76 & 1.20 \\
\hline $100 \% Q_{\mathrm{d}}$ & 34.48 & 4.44 & 4.30 & -3.15 & 57.32 & 60.41 & 3.09 \\
\hline $120 \% Q_{\mathrm{d}}$ & 41.40 & 3.92 & 3.83 & -2.30 & 56.20 & 57.90 & 1.70 \\
\hline
\end{tabular}

Table 6 Numerical results 
Table 7 Optimum scheme

\begin{tabular}{|c|c|c|c|c|c|c|c|c|c|c|c|}
\hline No. & $R_{0} / \mathrm{mm}$ & $R_{1} / \mathrm{mm}$ & $R_{2} / \mathrm{mm}$ & $T_{1} / \mathrm{deg}$ & $T_{2} / \mathrm{deg}$ & No. & $R_{0} / \mathrm{mm}$ & $R_{1} / \mathrm{mm}$ & $R_{2} / \mathrm{mm}$ & $T_{1} / \mathrm{deg}$ & $T_{2} / \mathrm{deg}$ \\
\hline 1 & 72.50 & 11.39 & 28.39 & 92.22 & 91.91 & 2 & 72.85 & 12.78 & 34.65 & 94.83 & 93.83 \\
\hline 3 & 73.20 & 10.00 & 31.87 & 94.39 & 92.96 & 4 & 73.54 & 13.83 & 30.13 & 92.87 & 94.00 \\
\hline 5 & 73.89 & 13.13 & 33.96 & 93.74 & 91.04 & 6 & 74.24 & 11.04 & 28.04 & 93.96 & 92.09 \\
\hline 7 & 74.59 & 10.52 & 27.70 & 95.91 & 93.48 & 8 & 74.93 & 12.43 & 31.52 & 95.26 & 93.65 \\
\hline 9 & 75.28 & 12.26 & 27.00 & 95.04 & 92.43 & 10 & 75.63 & 11.22 & 28.74 & 93.30 & 90.70 \\
\hline 11 & 75.98 & 13.65 & 30.83 & 96.35 & 92.61 & 12 & 76.33 & 10.35 & 32.22 & 95.70 & 93.30 \\
\hline 13 & 76.67 & 10.17 & 32.91 & 97.00 & 90.00 & 14 & 77.02 & 13.30 & 31.17 & 95.48 & 93.13 \\
\hline 15 & 77.37 & 12.61 & 33.61 & 94.17 & 91.39 & 16 & 77.72 & 11.57 & 29.78 & 92.00 & 90.52 \\
\hline 17 & 78.07 & 13.48 & 27.35 & 94.61 & 91.22 & 18 & 78.41 & 12.96 & 29.43 & 92.65 & 91.74 \\
\hline 19 & 78.76 & 14.00 & 34.30 & 96.13 & 90.87 & 20 & 79.11 & 11.91 & 29.09 & 93.52 & 92.78 \\
\hline 21 & 79.46 & 10.70 & 35.00 & 96.57 & 92.26 & 22 & 79.80 & 10.87 & 30.48 & 93.09 & 90.35 \\
\hline 23 & 80.15 & 11.74 & 33.26 & 92.43 & 91.57 & 24 & 80.50 & 12.09 & 32.57 & 96.78 & 90.17 \\
\hline
\end{tabular}

with the constraint conditions

$$
H_{i}(\boldsymbol{x})=c_{i}
$$

where $\boldsymbol{x}$ is a seven-dimensional vector, $\overline{\eta_{i}}$ is the weighted average efficiency of the three conditions with $i=1,2$, and $3, W_{i}$ is the weight factor of the three conditions, and $c_{i}$ is the head value of the three conditions.

Three weight factors are determined with the supertransitive approximation method which is proposed by Narasimhan [19].
Weight factor at $80 \% Q_{\mathrm{d}}, 100 \% Q_{\mathrm{d}}$, and $120 \% Q_{\mathrm{d}}$ is $0.286,0.428$, and 0.286 , respectively.

3.2.5 Multicondition Optimization Results. According to Eqs. (21) and (22), energy performance calculation program QE.exe is compiled. QE.exe is integrated by using Isight code. The adaptive simulated annealing algorithm is selected as the optimization algorithm, with major geometric parameters of the original design selected as the initial values, heads at three conditions as the

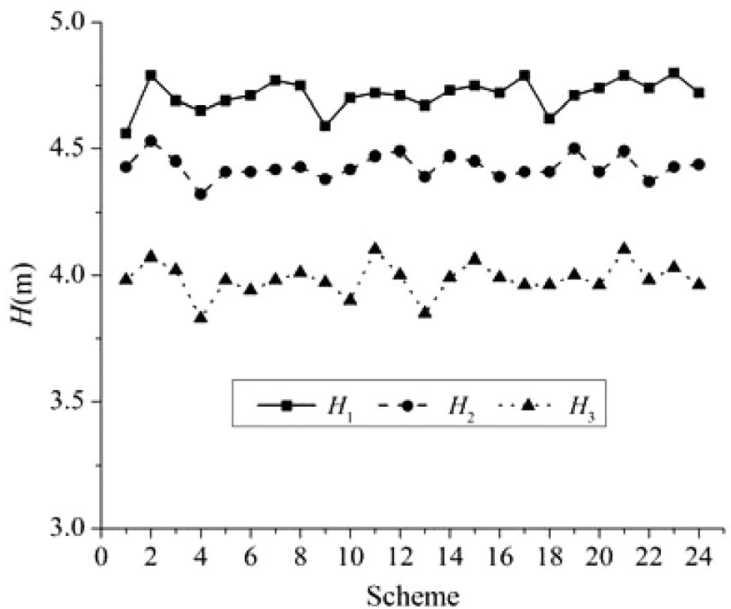

(a) $H$

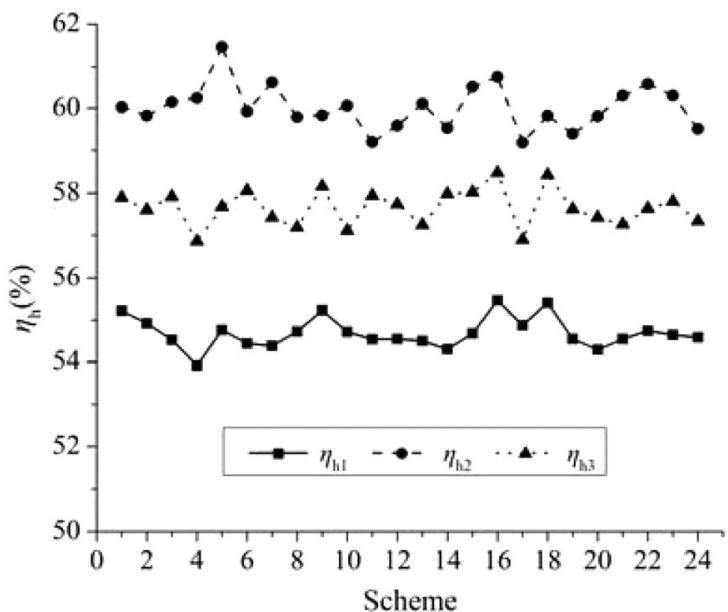

(c) $\eta_{\mathrm{h}}$

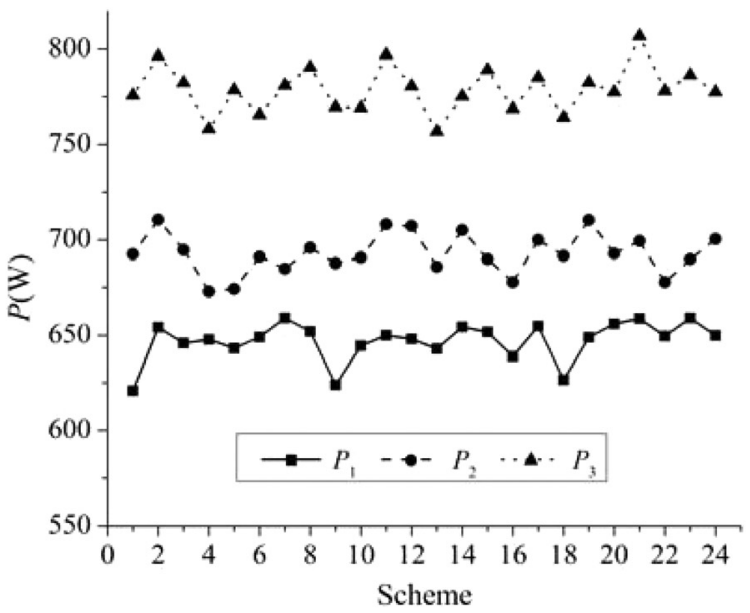

(b) $P$

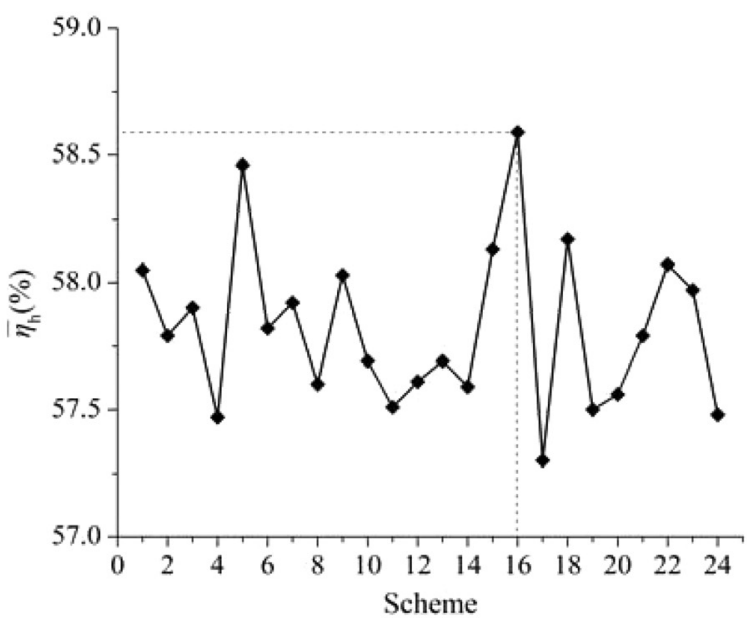

(d) $\bar{\eta}_{\mathrm{h}}$

Fig. 12 Optimization results 


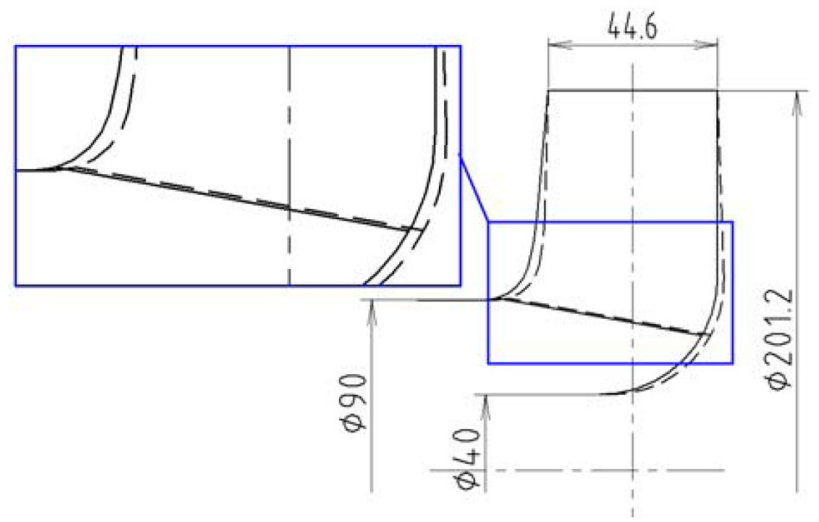

Fig. 13 The impeller meridional planes-before and after optimization

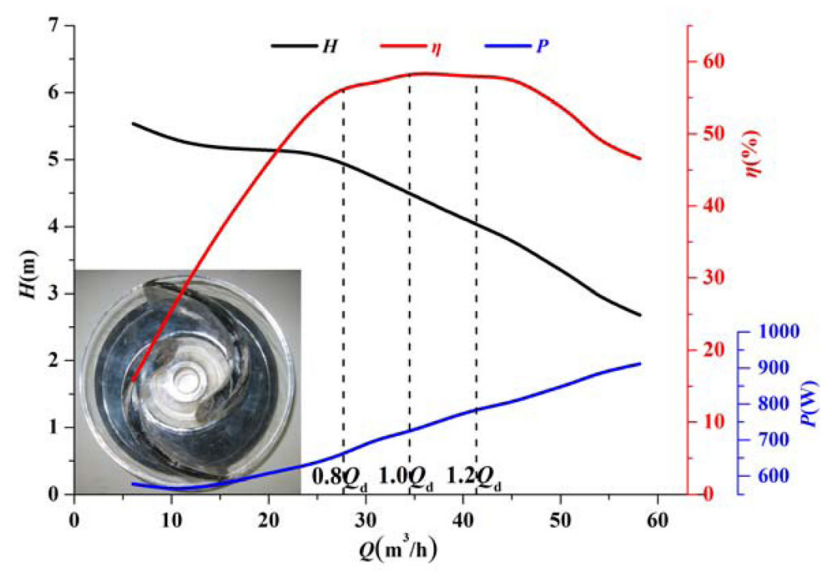

Fig. 14 Energy performance curves of optimized DBCP

Table 8 Numerical results

\begin{tabular}{|c|c|c|c|c|c|c|c|c|c|c|}
\hline & $H_{1} / \mathrm{m}$ & $\mathrm{H}_{2} / \mathrm{m}$ & $H_{3} / \mathrm{m}$ & $P_{1} / \mathrm{W}$ & $P_{2} / \mathrm{W}$ & $P_{3} / \mathrm{W}$ & $\eta_{h 1} / \%$ & $\eta_{h 2} / \%$ & $\eta_{h 3} / \%$ & $\bar{\eta}_{h} / \%$ \\
\hline Original & 4.75 & 4.30 & 3.83 & 639.52 & 668.1 & 745.53 & 55.76 & 60.41 & 57.9 & 58.36 \\
\hline Optimization & 4.81 & 4.44 & 4.06 & 648.11 & 677.97 & 780.02 & 55.72 & 61.47 & 58.66 & 59.02 \\
\hline
\end{tabular}

Table 9 Comparison of energy performances before and after optimization

\begin{tabular}{|c|c|c|c|c|c|c|c|c|c|}
\hline & \multirow[b]{2}{*}{$Q / \mathrm{m}^{3} \mathrm{~h}^{-1}$} & \multicolumn{2}{|c|}{$H / \mathrm{m}$} & \multicolumn{2}{|c|}{$P / \mathrm{W}$} & \multicolumn{2}{|c|}{$\eta / \%$} & \multicolumn{2}{|c|}{$\bar{\eta} / \%$} \\
\hline & & Original & Optimization & Original & Optimization & Original & Optimization & Original & Optimization \\
\hline $80 \% Q_{\mathrm{d}}$ & 27.58 & 4.91 & 4.96 & 675.66 & 658.81 & 54.56 & 56.32 & 56.23 & 57.69 \\
\hline $100 \% Q_{\mathrm{d}}$ & 34.48 & 4.44 & 4.50 & 727.06 & 722.78 & 57.37 & 58.48 & & \\
\hline $120 \% Q_{\mathrm{d}}$ & 41.40 & 3.92 & 4.03 & 785.97 & 785.99 & 56.20 & 57.89 & & \\
\hline
\end{tabular}

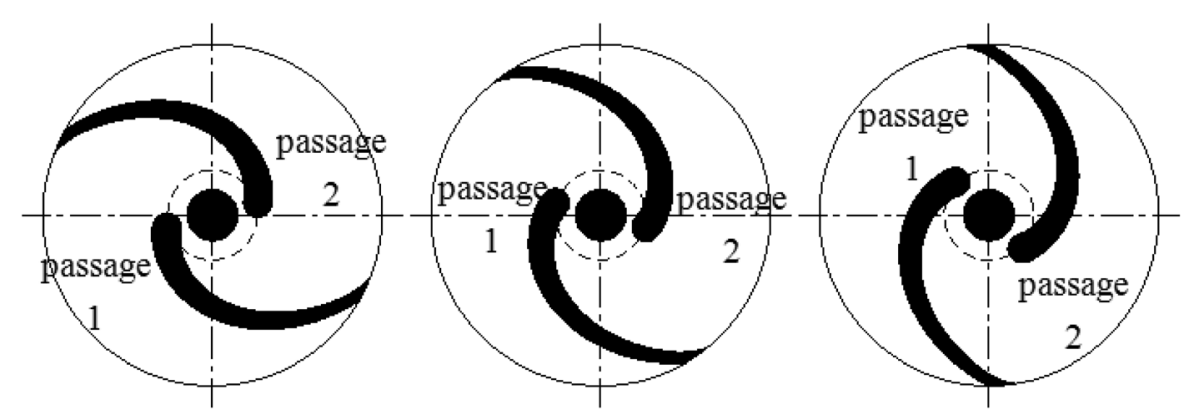
(a) $t=0$
(b) $t=T / 6$
(c) $t=T / 3$

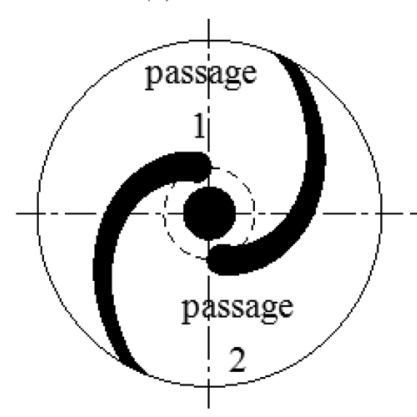

(d) $t=T / 2$

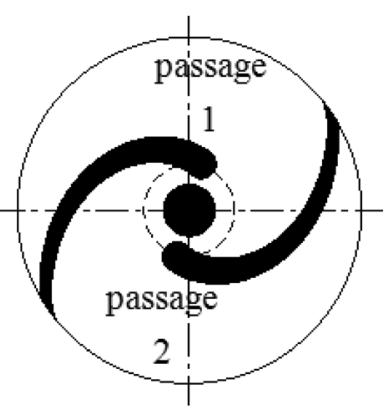

(e) $t=2 T / 3$

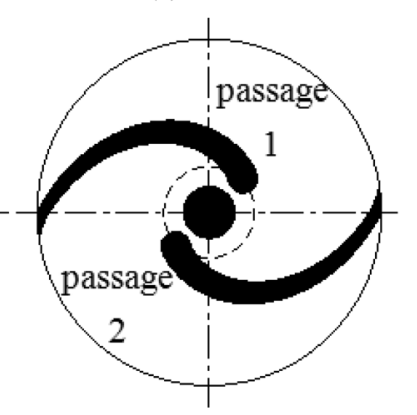

(f) $t=5 T / 6$

Fig. 15 Sketches of blades phase conditions 

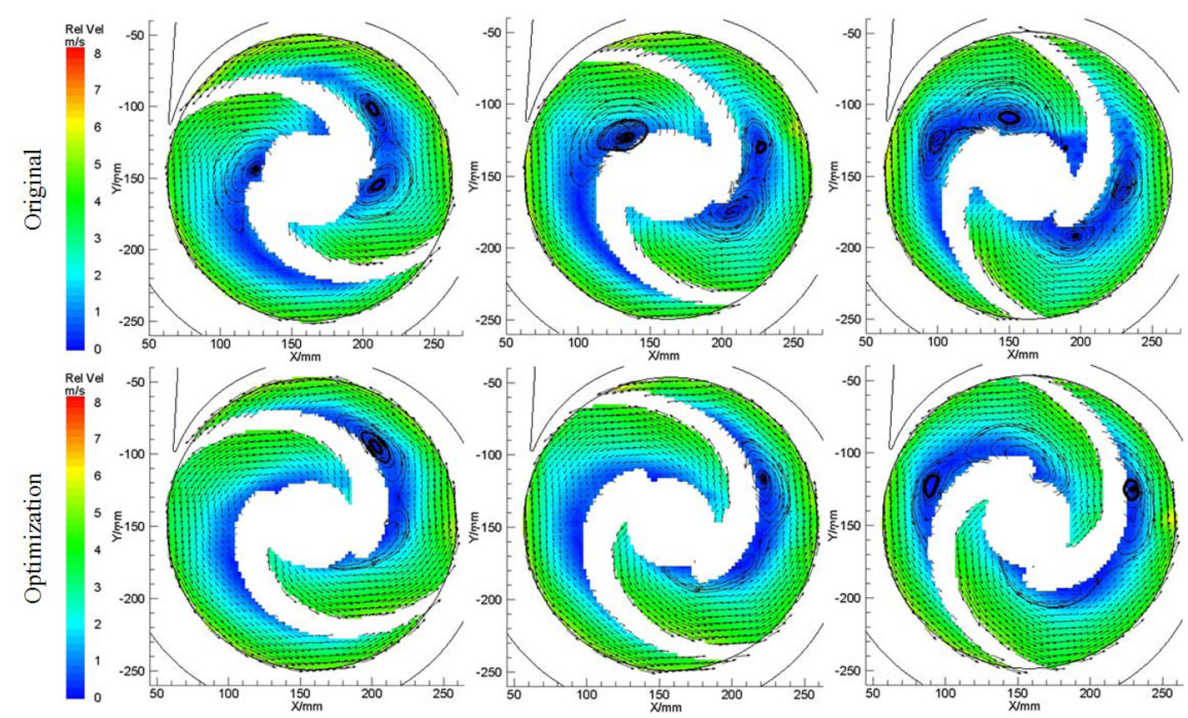

(a) $t=0$
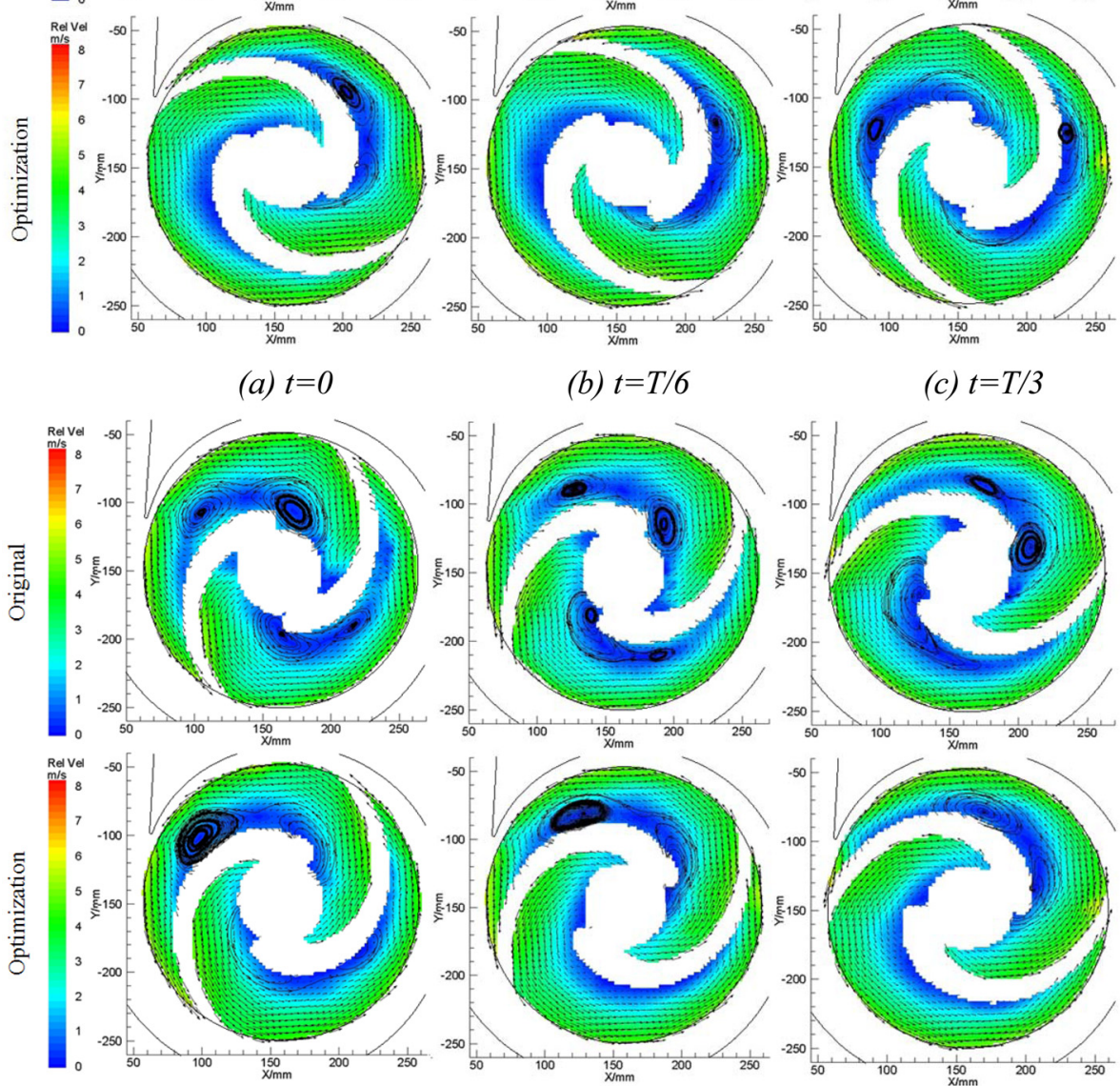

(b) $t=T / 6$

(c) $t=T / 3$

(d) $t=T / 2$
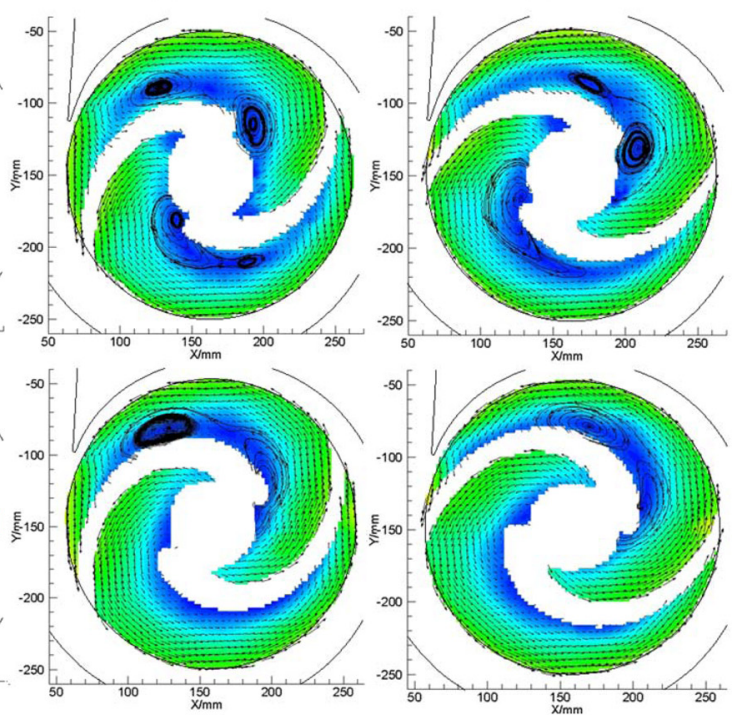

(e) $t=2 T / 3$

(f) $t=5 T / 6$

Fig. 16 Relative velocity distributions of impeller passage at $80 \% Q_{d}$

constraints conditions, and the maximum of weighted average efficiency at three conditions as the objective function.

The optimization results are shown in Table 3.

\subsection{Multicondition Numerical Optimization of Impeller} Meridional Plane

3.3.1 Numerical Simulation Method and Results. Based on impeller optimization results in Table 3 and the original design, Pro/E is selected to create impeller and TGrid is selected to divide mesh with Gambit. Grid numbers of semispiral suction chamber, impeller, and volute of scheme 2 are as shown in Table 4 and Fig. 10.

The flow through the DBCP was simulated with Fluent which uses the finite volume method to solve the Reynolds averaged Navier-Stokes equations for 3D incompressible steady flow. The flow model was complemented with the SST $k-\omega$ model. The mass-flow rate was specified at the inlet of the CFD domain (side entrance of semispiral suction chamber), and the outlet boundary was assumed to be outflow. All physical surfaces of the DBCP were set as no-slip wall and the near-wall regions were disposed with the standard wall functions method. The pressure-velocity coupling was calculated by means of the SIMPLE algorithm, and the convergence precision was set to $10^{-4}$.

Results of the grid independence test are shown in Table 5. It can be noted that when the total grid numbers are larger than $1.19 \times 10^{6}$, the head and the efficiency do not significantly change, where the relative error of head is $-3.15 \%$ and the absolute error of efficiency is $3.09 \%$.

Numerical errors at the three conditions are shown in Table 6. The maximum relative error of head is $3.26 \%$ at $80 \% Q_{\mathrm{d}}$ and the maximum absolute error of efficiency is $3.09 \%$ at $100 \% Q_{\mathrm{d}}$.

3.3.2 Numerical Optimization Model of the Impeller Meridional Plane. The impeller meridional plane of DBCP is shown in Fig. 11. The multicondition numerical optimization model is as follows.

Defining $\boldsymbol{x}=\left[R_{0}, R_{1}, R_{2}, T_{1}, T_{2}\right]^{\mathrm{T}}$ yields

$$
\bar{\eta}_{\mathrm{h}}=\frac{\sum \eta_{\mathrm{h} i}(\boldsymbol{x}) W_{i}}{\sum W_{i}} \rightarrow \max
$$




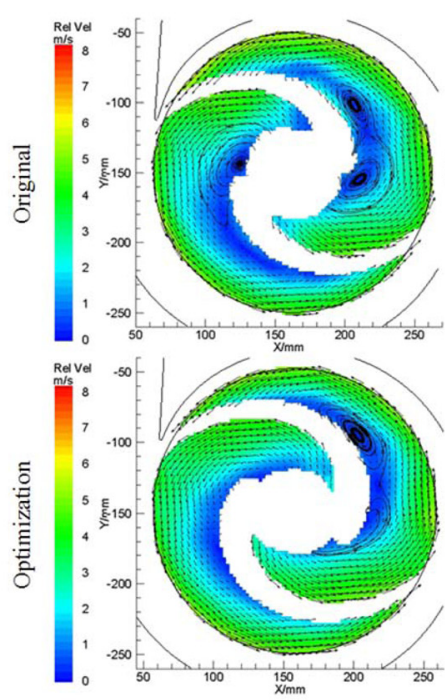

(a) $t=0$

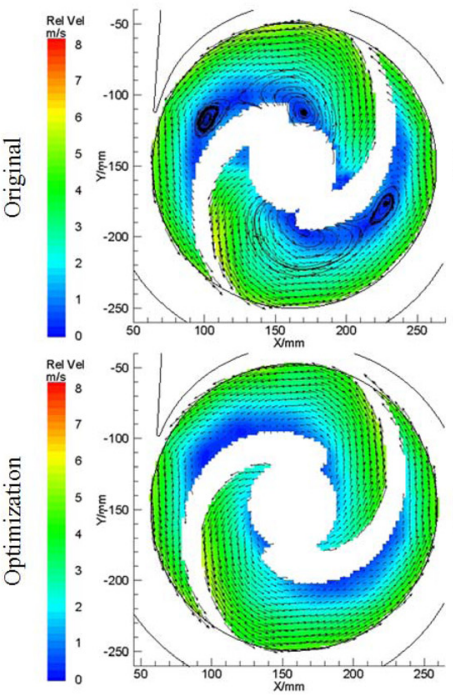

(d) $t=T / 2$
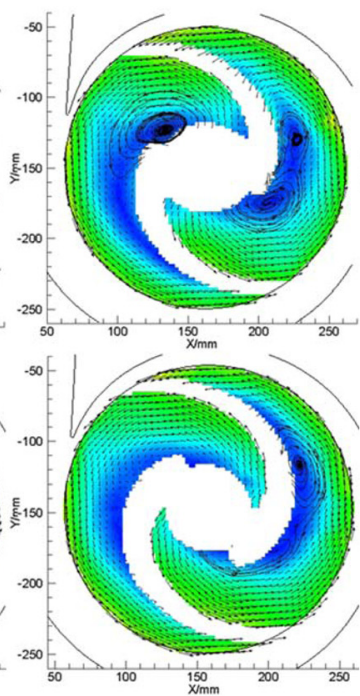

(b) $t=T / 6$
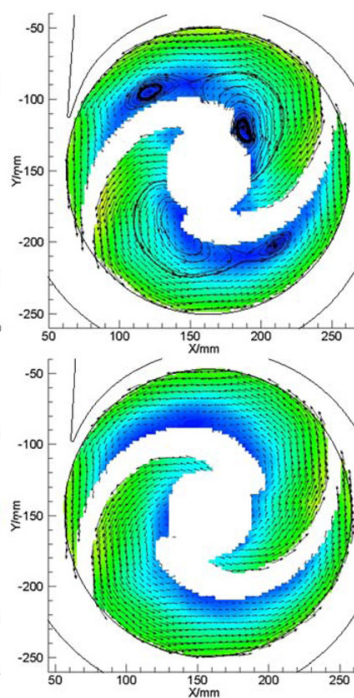

(e) $t=2 T / 3$
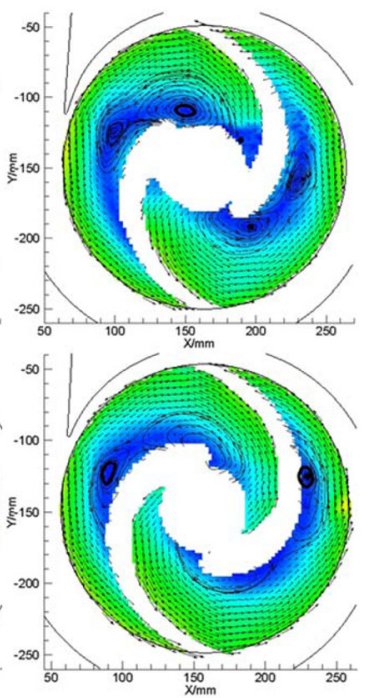

(c) $t=T / 3$
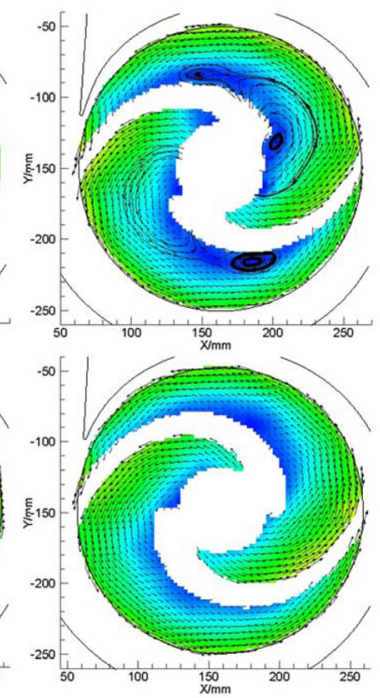

(f) $t=5 T / 6$

Fig. 17 Relative velocity distributions of impeller passage at $100 \% Q_{d}$

$$
\begin{gathered}
\eta_{\mathrm{h} i}=\rho g Q_{i} H_{i} / P_{i} \\
H_{i}=\Delta z+\frac{P_{\text {outlet }, i}-P_{\text {inlet }, i}}{\rho g} \\
P_{i}=N_{i} \frac{2 \pi n}{60}
\end{gathered}
$$

where $\boldsymbol{x}$ is a five-dimensional vector, $\bar{\eta}_{\mathrm{h}}$ is the weighted average hydraulic efficiency, $i$ is a design condition, $i=1,2, ., \eta_{\mathrm{h} i}$ is the hydraulic efficiency at $i$ condition, $W_{i}$ is the weight factor, $Q_{i}$ is the flow rate at $i$ condition, $H_{i}$ is the head at $i$ condition, $\Delta z$ is the head difference among the pump inlet and outlet, $P_{\text {inlet }, i}$ is the total pressure of the pump inlet at $i$ condition, $P_{\text {outlet }, i}$ is the total pressure of the pump outlet at $i$ condition, $P_{i}$ is the input power at $i$ condition without considering mechanics losses and clearance leakage, $N_{i}$ is the moment of the impeller at $i$ condition, and $n$ is the rotational speed.

3.3.3 Three-Condition Numerical Optimization of the Impeller Meridional Plane

Optimum scheme. Initial values of design variables are as follows: $R_{0}=76.5 \mathrm{~mm}, R_{1}=12 \mathrm{~mm}, R_{2}=31 \mathrm{~mm}, T_{1}=95 \mathrm{deg}$, and
$T_{2}=90 \mathrm{deg}$. The scopes of design variables are $R_{0} \in[72.5,80.5]$, $R_{1} \in[10,14], R_{2} \in[27,35], T_{1} \in[92,97]$, and $T_{2} \in[90,94]$. The optimum scheme was obtained with the optimal Latin hypercube $(\mathrm{OLH})$ method, as shown in Table 7.

The weight factors at $80 \% Q_{\mathrm{d}}, 100 \% Q_{\mathrm{d}}$, and $120 \% Q_{\mathrm{d}}$, as determined with the supertransitive approximation method, are 0.286 , 0.428 , and 0.286 , respectively.

Mesh generation and the numerical simulation method are the same as that above.

Optimization result. The calculations are performed on a Dell workstation with one processor (Windows XP, 64bit, 3.0 GHz, 4GB RAM) and the calculating time is about $196 \mathrm{~h}$ and $13 \mathrm{~min}$. Optimization results are shown in Fig. 12.

It is seen from Fig. 12(d) that weighted average hydraulic efficiency of 16 is the highest and reaches $58.59 \%$. Thus, the DBCP blades are redesigned based on 16. Figure 13 shows the impeller meridional planes of before and after optimization, in which the optimized meridional plane is indicated by the dotted lines.

TGrid is used to divide mesh with Gambit and the grid number of the impeller is 491,877 . The numerical simulation method is the same as that above. 
The numerical results before and after optimization are shown in Table 8 . From Table 8 we can see that heads, powers, and hydraulic efficiencies of the optimization impeller at the three conditions $\left(80 \% Q_{\mathrm{d}}, 100 \% Q_{\mathrm{d}}\right.$, and $\left.120 \% Q_{\mathrm{d}}\right)$ are larger than that of the original impeller. And three-condition weighted average efficiency increases from $58.36 \%$ to $59.02 \%$, which improved $0.66 \%$.

3.4 Energy Performance Test Verification. Figure 14 shows the energy performance curves of the optimized DBCP. The comparison of energy performances before and after optimization at $80 \% Q_{\mathrm{d}}, 100 \% Q_{\mathrm{d}}$, and $120 \% Q_{\mathrm{d}}$ are shown in Table 9. It is seen from Table 9 that the head of the optimized DBCP increased $0.05 \mathrm{~m}$ and the efficiency increased $1.76 \%$ at $80 \% Q_{\mathrm{d}}$. At $100 \% Q_{\mathrm{d}}$ the head of the optimized DBCP increased $0.06 \mathrm{~m}$ and the efficiency increased $1.11 \%$. At $120 \% Q_{\mathrm{d}}$ the head of optimized DBCP increased $0.11 \mathrm{~m}$ and the efficiency increased $1.69 \%$. And the three-condition weighted average efficiency of optimized DBCP increases from $56.23 \%$ to $57.69 \%$, which improved $1.46 \%$.

Test results show that the multicondition hydraulic optimization method of DBCP is feasible and could provide a certain reference for optimum design of other pumps.

\section{Internal Flow Fields Comparison Before and After Optimization}

In order to study the change of velocity field before and after optimization in the DBCP impeller, six different phase conditions (shown in Fig. 15) at $80 \% Q_{\mathrm{d}}, 100 \% Q_{\mathrm{d}}$, and $120 \% Q_{\mathrm{d}}$ are measured, that is, $t=0, T / 6, T / 3, T / 2,2 T / 3,5 T / 6$ with a phase interval being $30 \mathrm{deg}$. When $t=0, \theta$ is $28 \mathrm{deg}$ (shown in Fig. 4). The phase difference is set through the pulse delay time in the Insight $3 \mathrm{G}$ code and the pulse delay time is $5 \mathrm{~ms}$ if blades rotate $30 \mathrm{deg}$ at $n=1000 \mathrm{rpm}$.

Figure 16 represents the relative velocity distributions inside the original impeller passages at $80 \% Q_{\mathrm{d}}$. It can be seen from Fig. 16 that there are vortex regions in the two passages of the impeller. In passage 1 there is one vortex region at $t=0$ and $T / 6$ while there are two vortex regions at $t=T / 3, T / 2,2 T / 3$, and $5 T / 6$. In passage 2 , the two vortex regions decrease gradually and grow into one vortex at $t=5 T / 6$.

Also, Fig. 16 shows the relative velocity distributions of the optimized impeller passages at $80 \% Q_{\mathrm{d}}$. From Fig. 16 we can find that that there are vortex regions in the two passages of the impeller but the vortex regions in the optimized impeller are smaller

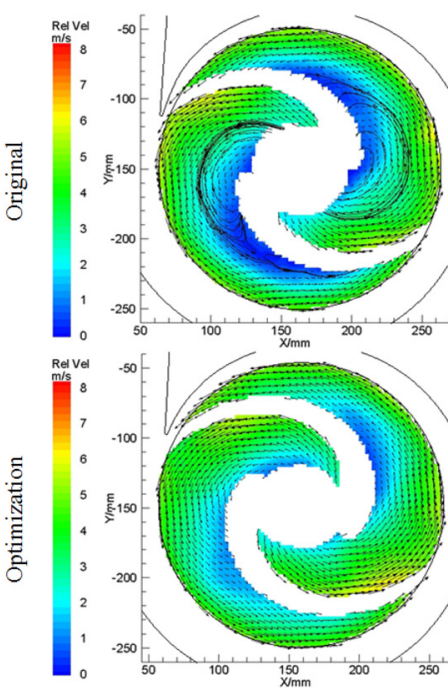

(a) $t=0$

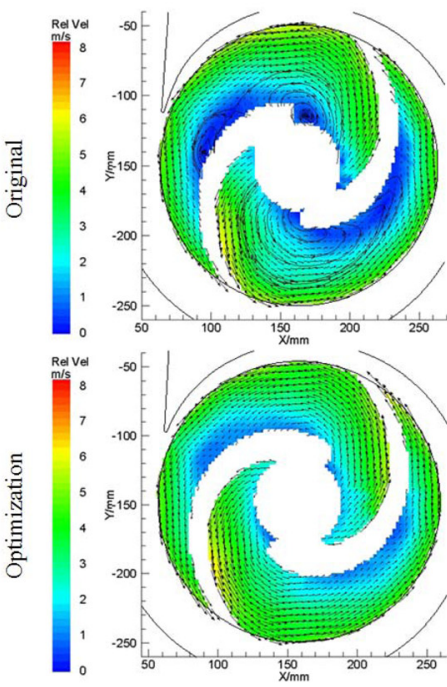

(d) $t=T / 2$

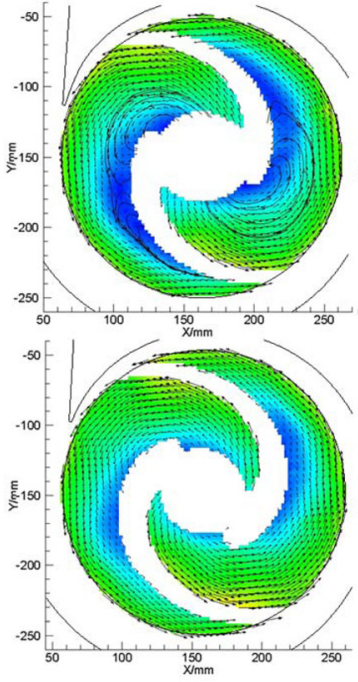

(b) $t=T / 6$
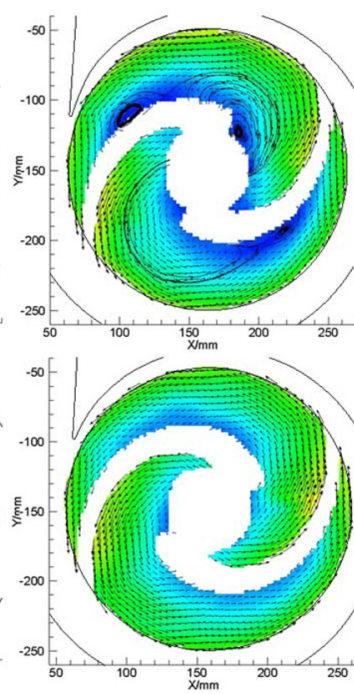

(e) $t=2 T / 3$

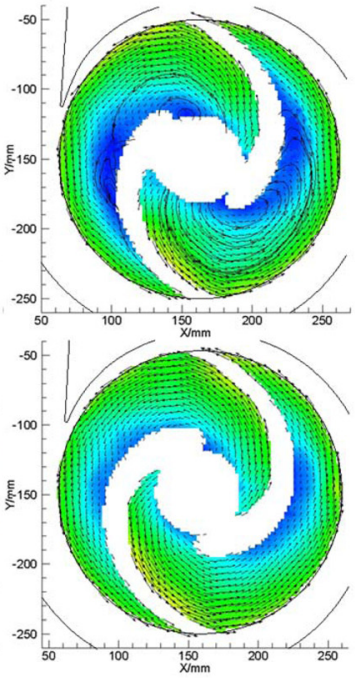

(c) $t=T / 3$
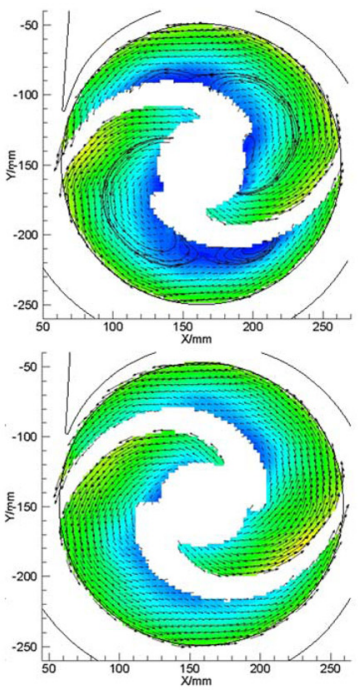

(f) $t=5 T / 6$

Fig. 18 Relative velocity distributions of impeller passage at $120 \% Q_{d}$ 
than those in the original impeller. In the impeller passage 1, the flow becomes smoother and there are no obvious vortex regions at $t=0$ and $T / 6$. From $t=T / 3$, there is one vortex region in passage 1 , which increases at first until its size reaches the maximum at $t=T / 2$ and thereafter decreases. Besides, the recirculation occurring in the inlet of the blade of impeller passage 1 from $t=T / 3$ decreases gradually. In impeller passage 2, the vortex region decreases gradually and disappears at $t=T / 2$.

Figure 17 illustrates the relative velocity distributions at $100 \% Q_{\mathrm{d}}$. As can be appreciated from Fig. 17, there are still vortex regions in the two passages of the impeller. In passage 1, initially $(t=0)$ there is a vortex region in the middle section of the blade and a recirculation region in the inlet of the blade. At $t=T / 6$ and $T / 3$ there is one vortex region in the inlet of the blade. While at $t=T / 2,2 T / 3$, and $5 T / 6$ there are two vortex regions in passage 1 . In passage 2 there is a small vortex at $t=0, T / 6$, and $T / 3$, while at $t=T / 2,2 T / 3$, and $5 T / 6$ there is a recirculation region in the inlet of the blade and a vortex region in the middle section of the blade.

Also, Fig. 17 shows the relative velocity distributions inside the optimized impeller at $100 \% Q_{\mathrm{d}}$. As compared to the original impeller, the optimized impeller has relatively smaller velocity regions near the middle section of the blade pressure side, which decrease gradually with the increase of flow rate, but there is better flow pattern and no obvious vortex region.

Figure 18 illustrates the relative velocity distributions at $120 \% Q_{\mathrm{d}}$. For the original impeller, there are also vortex regions in the two passages. In passage 1 , recirculation regions occur in the inlet of the blade at $t=0, T / 6$, and $T / 3$. There is a vortex region in the middle of the blade at $t=T / 6, T / 3$, and $T / 2$. While at $t=5 T / 6$ the vortex region in the middle of the blade disappears and a recirculation region occurs in the inlet of the blade. In passage 2 , a recirculation region in the inlet of the blade increases gradually along the blade toward the impeller outlet and there is a vortex region in the middle section of the blade at $t=2 T / 3$ and $5 T / 6$.

For the optimized impeller, there are low velocity regions near the middle section of the blade pressure side, which decrease gradually with the increase of flow rate, but the flow becomes smoother and there is no obvious vortex region.

\section{Conclusions}

As an attempt to optimize a two-blade DBCP, this paper selected major geometric parameters of original design as the initial values, heads at the three conditions as the constraints condition, and the maximum of weighted average efficiency at the three conditions as the objective function. An adaptive simulated annealing algorithm was used to solve the energy performance calculation model of DBCP. Based on the obtained results, threecondition automatic CFD optimization of the impeller meridional plane for the DBCP was realized by means of Isight software integrated Pro/E, Gambit, and Fluent software. The shroud arc radii $R_{0}$ and $R_{1}$, shroud angle $T_{1}$, hub arc radius $R_{2}$, and hub angle $T_{2}$ on the meridional plane were selected as the design variables and the maximum of weighted average hydraulic efficiency at the three conditions was chosen as the objective function. Wherein a numerical optimum scheme was obtained with the OLH method, weight factors were determined with the supertransitive approximation method. Performance characteristic test results show that the weighted average efficiency of the three-condition optimization has increased by $1.46 \%$ as compared to that of original design.

Unsteady internal flow of the DBCP impeller before and after optimization were measured with PIV technology. The experimental results show that unsteady flow (e.g., vortex and recirculation) in the impeller are considerably reduced under the three conditions (e.g., at $80 \% Q_{\mathrm{d}}, 100 \% Q_{\mathrm{d}}$, and $120 \% Q_{\mathrm{d}}$ ). At $80 \% Q_{\mathrm{d}}$ vortex regions always exist in the impeller both before and after optimization, but the vortex regions in the optimized impeller are obviously smaller than those in original impeller. At $100 \% Q_{\mathrm{d}}$ and $120 \% Q_{\mathrm{d}}$ there are vortex regions and recirculation regions in the original impeller, but in the optimized impeller the internal flow becomes considerably smoother and thus no obvious vortex regions or recirculation regions are observed.

\section{Acknowledgment}

The work was supported by the National Natural Science Fund of China (Grant Nos. 50825902, 51079062, 51179075, and 51209105), National Science \& Technology Pillar Program of China (Grant No. 2011BAF14B03), Natural Science Fund of Jiangsu Province of China (Grant Nos. BK2010346, BA2010155 and BY2011140), Science and Technology Support Program of Jiangsu Province of China (Grant Nos. BE2012129 and BE2012131), Six-Kinds-of-Top-Talent Program of Jiangsu Province of China, and Senior Professional Scientific Research Project of Jiangsu University of China (Grant No. 12JDG045).

\section{Nomenclature}

$Q=$ flow rate $\left(\mathrm{m}^{3} / \mathrm{h}\right)$

$Q_{\mathrm{d}}=$ flow rate of design condition $\left(\mathrm{m}^{3} / \mathrm{h}\right)$

$H=$ head (m)

$P=\operatorname{power}(\mathrm{W})$

$\eta=$ pump efficiency $(\%)$

$n=$ rotation speed $(\mathrm{rpm})$

$n_{\mathrm{s}}=$ specific speed $\left(\mathrm{m}^{3} / \mathrm{s}, \mathrm{m}, \mathrm{rpm}\right)$

$z=$ number of impeller blades

$\beta_{1}=$ blade inlet angle of impeller (deg)

$\beta_{2}=$ blade outlet angle of impeller (deg)

$\varphi=$ blade wrap angle of impeller (deg)

$b_{2}=$ outlet blade width of impeller (mm)

$b_{3}=$ volute inlet width $(\mathrm{mm})$

$D_{1}=$ impeller inlet diameter $(\mathrm{mm})$

$D_{2}=$ impeller outlet diameter $(\mathrm{mm})$

$D_{3}=$ volute inlet diameter $(\mathrm{mm})$

$q_{1}=$ leakage flow rate through sealing ring at impeller inlet $\left(\mathrm{m}^{3} / \mathrm{h}\right)$

$f=$ sectional area of sealing gap $\left(\mathrm{mm}^{2}\right)$

$R_{\mathrm{m}}=$ radius of sealing ring $(\mathrm{mm})$

$b=$ sealing gap width $(\mathrm{mm})$

$\mu=$ velocity coefficient of sealing gap

$\zeta=$ coefficient of fillet radius

$\lambda=$ hydraulic resistance coefficient

$l=$ sealing gap length $(\mathrm{mm})$

$\Delta H_{\mathrm{m}}=$ pressure difference over the seal (m)

$\eta_{\mathrm{v}}=$ volumetric efficiency $(\%)$

$\sigma=$ Stodola slip factor

$\Psi_{2}=$ blockage coefficient of blade outlet

$S_{u 2}=$ circumference thickness of blade outlet $(\mathrm{mm})$

$\Delta h_{i}=$ hydraulic losses $(\mathrm{m})$

$j=1,2,3, \ldots \ldots, 7$

$k_{j}=$ loss coefficient

$\lambda_{1}=$ hydraulic resistance coefficient

$l_{1 \mathrm{a}}=$ length of impeller passage $(\mathrm{mm})$

$v_{1 \mathrm{a}}=$ average velocity in impeller inlet area and outlet area $(\mathrm{m} / \mathrm{s})$

$D_{1 \mathrm{a}}=$ hydraulic equivalent diameter of impeller $(\mathrm{mm})$

$\delta_{1}=$ surface roughness of impeller $(\mathrm{m})$

$v=$ inlet velocity of impeller $(\mathrm{m} / \mathrm{s})$

$v_{\mathrm{s}}=$ average velocity of volute throat $(\mathrm{m} / \mathrm{s})$

$\lambda_{2}=$ hydraulic resistance coefficient

$l_{2 \mathrm{a}}=$ length of volute passage $(\mathrm{mm})$

$v_{\text {th }}=$ average velocity of volute $(\mathrm{m} / \mathrm{s})$

$D_{2 \mathrm{a}}=$ hydraulic equivalent diameter of volute passage $(\mathrm{mm})$

$\varphi_{0}=$ volute tongue angle (deg)

$\delta_{2}=$ surface roughness of volute $(\mathrm{m})$

$\Delta h=$ total hydraulic losses in pump (m)

$\eta_{\mathrm{h}}=$ hydraulic efficiency $(\%)$

$\Delta P_{1}=$ mechanical friction losses in the bearing and stuffing box $(\mathrm{W})$ 
$\Delta P_{2}=$ Frick friction loss $(\mathrm{W})$

$e=$ thickness of impeller shroud and hub (mm)

$\eta_{\mathrm{m}}=$ mechanical efficiency $(\%)$

$\xi_{k}=$ correct coefficient

$k=0,1$, 10

$\boldsymbol{x}=$ vector

$\overline{\eta_{i}}=$ weighted average efficiency of $i$ conditions $(\%)$

$W_{i}=$ weight factor of $i$ conditions

$c_{i}=$ head value of $i$ conditions (m)

$i=1,2,3$

$R_{0}=$ impeller shroud arc radius $(\mathrm{mm})$

$R_{1}=$ impeller shroud arc radius (mm)

$R_{2}=$ impeller hub arc radius $(\mathrm{mm})$

$T_{1}=$ impeller shroud angle $(\mathrm{deg})$

$T_{2}=$ impeller hub angle (deg)

$\bar{\eta}_{\mathrm{h}}=$ weighted average hydraulic efficiency of $i$ conditions (\%)

$\eta_{\mathrm{h} i}=$ hydraulic efficiency at $i$ condition (\%)

$Q_{i}=$ flow rate at $i$ condition $\left(\mathrm{m}^{3} / \mathrm{h}\right)$

$H_{i}=$ head at $i$ condition (m)

$\Delta z=$ head difference among pump inlet and outlet (m)

$P_{\text {inlet }, i}=$ total pressure of pump inlet at $i$ condition $(\mathrm{Pa})$

$P_{\text {outlet }, i}=$ total pressure of pump outlet at $i$ condition $(\mathrm{Pa})$

$P_{i}=$ input power at $i$ condition without considering mechanics losses and clearance leakage (W)

$N_{i}=$ moment of impeller at $i$ condition $(\mathrm{N} \mathrm{m})$

\section{References}

[1] Karassik, I. J., Messina, J. P., Cooper, P., and Heald, C. C., 2008, Pump Handbook, 4th ed., McGraw-Hill, New York.

[2] Guan, X. F., 2011, Modern Pump Theory and Design, China Astronautic, Beijing, China.

[3] Liu, H. L., and Tan, M. G., 2012, Double Channel Pump, Jiangsu University Press, Zhenjiang, China.
[4] Chen, X., and Zhang, F. Y., 2000, "Design of the Closed Double Blade Pump," Pump Technol., 3, pp. 19-23.

[5] Huang, J. G., 2003, "Design and Performance Characteristic of Impeller for Sewage Pump," Pump Technol., 2, pp. 17-21.

[6] Zhao, W. Y., Wang, Z., Li, Y. S., and Zhang, L., 2008, "Numerical Simulation of Impeller Wear for a Centrifugal Sewage Pump," Pump Technol., 3, pp. 17-19.

[7] Zhu, R. S., Hu, Z. Q., and Yang, A. L., 2010, "Optimum Design of Efficient Sewage Pump Impeller," Pump Technol., 3, pp. 5-7.

[8] Zhu, R. S., Hu, Z. Q., and Yang, A. L., 2011, "Numerical Simulation of Unsteady Flow in Double-Blade Pump," J. Drain. Irrig. Machin. Eng., 29(1), pp. 26-30.

[9] Zhu, R. S., Hu, Z. Q., and Fu, Q., 2010, "Numerical Simulation of Pressure Fluctuation in Double-Blade Pump," Trans. CSAE, 26(6), pp. 129-134.

[10] Liu, H. L., Wang, K., Yuan, S. Q., Tan, M. G., Wang, Y., and Ru, W. M., 2012, "3D PIV Test of Inner Flow in a Double Blade Pump Impeller," Chin. J. Mech. Eng., 25(3), pp. 491-497.

[11] Wang, K., Liu, H. L., Yuan, S. Q., Tan, M. G., and Yang, D. S., 2011, “3-D PIV Test of Inner Flow in a Double-Blade Pump Under Zero Flow Rate Condition," Trans. Chin. Soc. Agr. Machin., 42(7), pp. 75-79.

[12] Tan, L., Cao, S. L., Wang, Y. M., and Zhu, B. S., 2012, "Numerical Simulation of Cavitation in a Centrifugal Pump at Low Flow Rate," Chin. Phys. Lett., 29(1), p. 014702

[13] Lucius, A., and Brenner, G., 2011, "Numerical Simulation and Evaluation of Velocity Fluctuations During Rotating Stall of a Centrifugal Pump," ASME J. Fluids Eng., 133(8), p. 081102.

[14] Li, Z. F., Wu, D. Z., Wang, L. Q., and Huang, B., 2010, "Numerical Simulation of the Transient Flow in a Centrifugal Pump During Starting Period," ASME J. Fluid Eng., 132(8), p. 081102.

[15] González, J., Fernández, J., Blanco, E., and Santolaria, C., 2002, "Numerical Simulation of the Dynamic Effects Due to Impeller-Volute Interaction in a Centrifugal Pump," ASME J. Fluids Eng., 124(2), pp. 348-355.

[16] Wu, Y. L., Liu, S. H., Yuan, H. J., and Shao, J., 2011, "PIV Measurement on Internal Instantaneous Flows of a Centrifugal Pump," Sci. China Technol. Sci., 54(2), pp. 270-276.

[17] Westra, R. W., Broersma, L., van Andel, K., and Kruyt, N. P., 2010, "PIV Measurements and CFD Computations of Secondary Flow in a Centrifugal Pump Impeller," ASME J. Fluids Eng., 132(6), p. 061104.

[18] Benra, F.-K., Dohmen, H. J., and Sommer, M., 2006, "Flow Field Visualization of a Single-Blade Centrifugal Pump Using PIV-Method-Comparison to Numerical Results," J. Visual., 9(4), pp. 358-358.

[19] Narasimhan, R., 1982, "A Geometric Averaging Procedure for Constructing Supertransitive Approximation to Binary Comparison Matrices," Fuzzy Sets Syst., 8(1), pp. 53-61. 